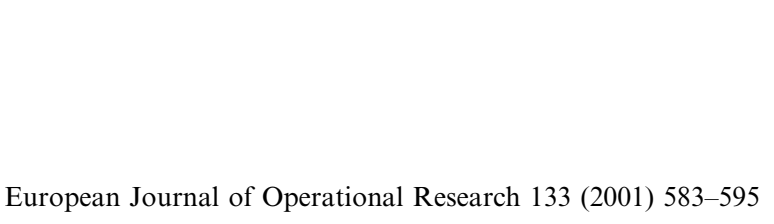

\title{
An efficient mean field approach to the set covering problem
}

\author{
Mattias Ohlsson *, Carsten Peterson, Bo Söderberg \\ Complex Systems Division, Department of Theoretical Physics, University of Lund, Sölvegatan 14A, S-223 62 Lund, Sweden
}

Received 23 March 1999; accepted 20 July 2000

\begin{abstract}
A mean field feedback artificial neural network (ANN) algorithm is developed and explored for the set covering problem. A convenient encoding of the inequality constraints is achieved by means of a multilinear penalty function. An approximate energy minimum is obtained by iterating a set of mean field equations, in combination with annealing. The approach is numerically tested against a set of publicly available test problems with sizes ranging up to $5 \times 10^{3}$ rows and $10^{6}$ columns. When comparing the performance with exact results for sizes where these are available, the approach yields results within a few percent from the optimal solutions. Comparisons with other approximate methods also come out well, in particular given the very low CPU consumption required - typically a few seconds. Arbitrary problems can be processed using the algorithm via a public domain server. (c) 2001 Elsevier Science B.V. All rights reserved.
\end{abstract}

Keywords: Neural networks; Mean field annealing; Set covering; Combinatorial optimization

\section{Introduction}

The set covering problem (SCP) is a wellknown NP-hard combinatorial optimization problem, which represents many real-world resource allocation problems. Exact solutions can be obtained by, e.g., a branch-and-bound approach for modestly sized problems. For larger problems various approximative schemes have been suggested (see, e.g., [1,2]). In this paper we develop a

\footnotetext{
* Corresponding author. Tel.: +46 4622277 82; fax: +46 46 2229686.

E-mail addresses: mattias@thep.lu.se (M. Ohlsson), carsten@thep.lu.se (C. Peterson), bs@thep.lu.se (B. Söderberg). Web.: http://www.thep.lu.se/tf2/complex/
}

novel approach based on feedback artificial neural networks (ANN), derived from the mean field approximation to the thermodynamics of spin systems.

ANN is a computer paradigm that has gained a lot of attention during the last 5-10 years. Most of the activities have been directed towards feed-forward architectures for pattern recognition or function approximation. ANN, in particular feedback networks, can also be used for difficult combinatorial optimization problems (e.g., [6-11]). Here ANN introduces a new method that, in contrast to most existing search and heuristics techniques, is not based on exploratory search to find the optimal configuration. Rather, the neural units find their way in a fuzzy manner through an interpolating, continuous space towards good 
solutions. There is a close connection between feedback ANN and spin systems in statistical physics. Consequently, many mathematical tools used for dealing with spin systems can be applied to feedback ANN. Two steps are involved when using ANN for combinatorial optimization:

1. Map the problem onto an energy function, e.g.,

$$
E(\mathscr{S})=\frac{1}{2} \sum_{i j} w_{i j} s_{i} s_{j},
$$

where $\mathscr{S}=\left\{s_{i} ; i=1 \ldots N\right\}$ is a set of binary spin variables $s_{i} \in\{0,1\}$, representing the elementary choices involved in minimizing $E$, while the weights $w_{i j}$ encode the costs and constraints.

2. To find configurations with low $E$, iterate the mean field (MF) equations

$$
v_{i}=1 /\left(1+\exp \left(\frac{1}{T} \frac{\partial E(\mathscr{V})}{\partial v_{i}}\right)\right),
$$

where $T$ is a fictitious temperature while $\mathscr{V}=$ $\left\{v_{i}\right\}$, where $v_{i} \in[0,1]$ represents the thermal average $\left\langle s_{i}\right\rangle_{T}$, and allows for a probabilistic interpretation.

Eqs. (1) and (2) only represent one example. More elaborate encodings have been considered, e.g., based on Potts spins allowing for more general basic decisions elements than simple binary ones [17]. A propagator formalism based on Potts neurons has been developed for handling topological complications in, e.g., routing problems $[7,10]$.

The ANN approach for SCP that we develop here differs from the one that was successfully used for the somewhat related knapsack problem in $[13,14]$, in particular with respect to encoding the constraints. Whereas a non-linear step-function was used in $[13,14]$, we will here use a multilinear penalty, which in addition to being theoretically more appealing, also appears to be very efficient. Furthermore, an automatic procedure for setting the relevant $T$-scale is devised.

The algorithm is extensively tested against a set of publicly available benchmark problems [15] with sizes (rows $\times$ columns) ranging from $200 \times$ 1000 to $5000 \times 10^{6}$. The approach yields results, typically within a few percent from the exact op- timal solutions, for sizes where these are available. Comparisons with other approximate methods also come out well. The algorithm is extremely rapid - the typical CPU demand is only a few seconds (on a $400 \mathrm{MHz}$ Pentium II).

A public domain WWW server has been set up, where arbitrary problems can be solved interactively.

This paper is organized as follows: In Section 2 we define the SCP, and in Section 3 we describe its encoding in terms of a neural network energy function and discuss the MF treatment. Section 4 contains numerical explorations and comparisons. A brief summary is given in Section 5. Appendices $\mathrm{A}$ and $\mathrm{B}$ contain a derivation of the mean field equations, and some algorithmic implementation issues, respectively. Tables from the numerical explorations are found in Appendix C, while Appendix D contains pointers and instructions for the WWW server. It should be stressed that this paper is self-contained - no prior knowledge of feedback neural networks or the MF approximation is necessary.

\section{The set covering problem}

The SCP is the problem of finding a subset of the columns of an $M \times N$ zero-one matrix

$A=\left\{a_{k i} \in\{0,1\} ; k=1, \ldots, M ; i=1, \ldots, N\right\}$

that covers all rows at a minimum cost, based on a set of column costs $\left\{c_{i} ; i=1, \ldots, N\right\}$. SCP is conveniently described using a set of binary variables, $\mathscr{S}=\left\{s_{i} \in\{0,1\} ; i=1, \ldots, N\right\}$. More precisely, $\mathrm{SCP}$ is defined as follows:

Minimize $\sum_{i=1}^{N} c_{i} s_{i}$

subject to $\sum_{i=1}^{N} a_{k i} s_{i} \geqslant 1, \quad k=1, \ldots, M$,

with

$$
s_{i} \in\{0,1\}, \quad i=1, \ldots, N .
$$

Eq. (4) states that at least one column must cover a particular row. The special case where all $\operatorname{costs} c_{i}$ are equal is called the unicost SCP. There is sub- 
class of SCPs that has a nice graphical interpretation: If the zero-one matrix $A$ has the property that each row contains exactly two 1's then we can interpret $A$ as the vertex-edge matrix of a graph with $N$ vertices and $M$ edges. The unicost SCP is then a vertex covering problem, where the task is to find the minimal number of vertices that covers all edges of the graph. SCPs (including weighted vertex covering) are NP-hard combinatorial optimization problems. If the inequalities of Eq. (4) are replaced by equalities, one has the set partition problem (SPP). Both SCP and SPP have numerous resource allocation applications.

The following quantities will be used later:

Density: $\quad \rho=\frac{1}{M N} \sum_{k i} a_{k i}$,

Column sums: $\quad \hat{N}_{i}=\sum_{k}^{M} a_{k i}$,

Row sums: $\quad \hat{M}_{k}=\sum_{i}^{N} a_{k i}$.

\section{The mean field approach}

\subsection{The energy function for $S C P$}

We start by mapping the SCP of Eqs. (3)-(5) onto a spin energy function $E(\mathscr{S})$ (step 1 in Section 1):

$E(\mathscr{S})=\sum_{i=1}^{N} c_{i} s_{i}+\alpha \sum_{k=1}^{M} \prod_{i=1}^{N}\left(1-a_{k i} s_{i}\right)$.

The first term yields the total cost and the second one represents the covering constraint of Eq. (4) by imposing a penalty if a row is not covered by any column.

The constraint term is a multilinear polynomial in the spin variables $s_{i}$, i.e., it is a linear combination of products $s_{1} s_{2} \cdots s_{K}$ of distinct spins. This is attractive from a theoretical point of view, and the differentiability of $E$ enables a more quantitative analysis of the dynamics of the MF algorithm.

An alternative would be to implement the inequality constraints using a piecewise linear function [14], $\alpha \sum_{k=1}^{M} \phi\left(1-\sum_{i=1}^{N} a_{k i} s_{i}\right)$

with $\phi(x)=x \Theta(x)=x$ if $x>0$ and 0 otherwise. This yields a non-differentiable energy function and generally an inferior performance as compared to the polynomial representation (9).

\subsection{The statistical mechanics framework}

The next step is to minimize $E(\mathscr{S})$. Using some local updating rule, e.g., $s_{i} \rightarrow-s_{i}$ if the corresponding change in energy is negative, will most often yield a local minimum close to the starting point, with poor solutions as a result. Simulated annealing (SA) [9] is one way of escaping from local minima since it allows for uphill moves in $E$. In SA a sequence of configurations $\mathscr{S}$ is generated according to a stochastic algorithm, such as to emulate the probability distribution

$P(\mathscr{S})=\frac{\mathrm{e}^{-E(\mathscr{S}) / T}}{\sum_{\mathscr{S}^{\prime}} \mathrm{e}^{-E(\mathscr{S}) / T}}$,

where the sum runs over all possible configurations $\mathscr{S}^{\prime}$. The parameter $T$ (temperature) acts as a noise parameter. For large $T$ the system will fluctuate heavily since $P(\mathscr{S})$ is very flat. For the SCP this implies that the sequence contains mostly poor and infeasible solutions. On the other hand, for a small $T, P(\mathscr{S})$ will be narrow, and the sequence will be strongly dependent upon the initial configuration and contain configurations only from a small neighborhood around the initial point. In SA one generates configurations while lowering $T$ (annealing), thereby diminishing the risk of ending up in a suboptimal local minimum. This is quite CPU-consuming, since one has to generate many configurations for each temperature following a careful annealing schedule (typically $T_{k}=T_{0} /$ $\log (1+k)$ for some $\left.T_{0}\right)$ in order to be certain to find the global minimum.

In the MF approach the costly stochastic SA is approximated by a deterministic process. MF also contains an annealing procedure. The original binary variables $s_{i}$ are replaced by continuous MF 
variables $v_{i} \in[0,1]$, with a dynamics given by iteratively solving of the MF equations for each $T$.

An additional advantage of the MF approach is that the continuous MF variables can evolve in a space not accessible to the original variables. The intermediate configurations at non-zero $T$ have a natural probabilistic interpretation.

\subsection{MF theory equations}

Our objective is now to minimize $E$ using the MF method. The binary spin variables $s_{i}$ are replaced by $\mathrm{MF}$ variables $v_{i}$, representing solutions to the MF equations (a derivation of these is given in Appendix A).

$$
\begin{aligned}
v_{i} & =1 /\left(1+\exp \left(\frac{1}{T} \Delta E_{i}\left(\mathscr{V}^{(i)}\right)\right)\right), \\
i & =1, \ldots, N,
\end{aligned}
$$

where

$$
\begin{aligned}
\Delta E_{i}\left(\mathscr{V}^{(i)}\right) & =E\left(\mathscr{V}^{(i)}, v_{i}=1\right)-E\left(\mathscr{V}^{(i)}, v_{i}=0\right) \\
& =\partial E / \partial v_{i} .
\end{aligned}
$$

The set $\mathscr{V}^{(i)}$ denotes the complementary set $\left\{v_{j}, j \neq i\right\}$. From Eq. (9) we get

$$
\Delta E_{i}\left(\mathscr{V}^{(i)}\right)=c_{i}-\alpha \sum_{k=1}^{M} a_{k i} \prod_{j \neq i}^{N}\left(1-a_{k j} v_{j}\right) .
$$

The MF equations are solved iteratively while annealing in $T$. It should be noted that the equation for $v_{i}$ does not contain any feedback, i.e., no explicit dependence on $v_{i}$ itself. This yields a smooth convergence, and it is usually only necessary with a few iterations at each value of $T$. What remains to be specified are the parameters $\alpha$ and $T$. The latter will be discussed next and we return to the choice of $\alpha$ in connection with the numerical explorations in Section 4.

\subsection{Critical temperatures}

In the limit of high temperatures, $T \rightarrow \infty$, the MF variables $\left\{v_{i}\right\}$ will, under the dynamics defined by iteration of Eq. (12), converge to a trivial symmetric fixed point with $v_{i}=1 /(1+\exp (0))=\frac{1}{2}$, corresponding to no decision taken. At a finite but high $T$, the corresponding fixed point will typically deviate slightly from the symmetric point.

For many problems, a bifurcation occurs (indicative of a transition from a disordered phase to an ordered one) at a critical temperature $T_{\mathrm{c}}$, where the trivial fixed point loses stability and other fixed points emerge, which as $T \rightarrow 0$ converge towards definitive candidate solutions to the problem, in terms of $v_{i} \in\{0,1\}$. For some problems, a cascade of bifurcations occur, each at a distinct critical temperature, but in the typical case there is a single bifurcation.

It is then of interest to estimate the position of $T_{\mathrm{c}}$, which defines a suitable starting point for the MF algorithm. Such an estimate can be obtained by means of a linear stability analysis for the dynamics close to the fixed point. For the special case of a symmetric unicost SCP with constant row and column sums for the matrix $A, T_{\mathrm{c}}$ can be found as the largest eigenvalue of the matrix

$$
\alpha 2^{-\rho N}\left(\delta_{i j}-1\right) \sum_{k} a_{k i} a_{k j} \text {. }
$$

Estimation of the largest eigenvalue gives

$T_{\mathrm{c}} \approx \alpha \rho^{2} M 2^{-\rho N}$,

where $\rho$ is defined in (6).

For non-unicost problems, $T_{\mathrm{c}}$ is harder to estimate, and there might even be no bifurcation at all. For such problems, a suitable initial $T$ is instead determined by means of a fast preliminary run of the algorithm (see below).

\section{Numerical explorations}

\subsection{Implementation details}

The annealing schedule for $T$ and the value of the constraint parameter $\alpha$ have to be determined before we can run the algorithm. The former is accomplished by a geometric decrease of $T$ :

$T_{t+1}=k T_{t}$, 
where $k$ is set to 0.80 and $T_{0}$ is determined by a fast prerun of the algorithm (see below). The parameter $k$ determines how fast the temperature is lowered. Too rapid decrease of $T$ will result in poor solutions and the value 0.80 was found (numerically) to be a good compromise between speed and solution quality. The number of iterations of Eqs. (12) for each value of $T$ is not fixed; $T$ is lowered only when all $v_{i}$ have converged.

In order to ensure a valid solution at low $T$, the size of the constraint term in Eq. (14) must be larger than the largest $\cos t c_{\max }$ that is part of the solution. Using a too large $\alpha$ will however reduce the solution quality since $E$ is then dominated by the covering constraint. Our choice of $\alpha$ therefore depends on $c_{\max }$. Ideally, $M /(\rho M)=1 / \rho$ columns would suffice to cover each row of $A$. If we further optimistically assume that the $1 / \rho$ smallest costs can be chosen for the solution, $c_{\max }$ can easily be found. However, for most problems we need more than $1 / \rho$ columns, which makes it difficult to estimate $c_{\max }$ except for unicost problems where all column costs are equal.

To determine (an approximate) $c_{\max }$ for a nonunicost SCP, we perform a fast prerun with a smaller annealing factor $k=0.65$, and with $\alpha=$ 1.01. From this prerun one can also obtain an estimate of the critical temperature $T_{\mathrm{c}}$ as the $T$ where the saturation (defined below) deviates from 0 . The second run is then initated at $T_{0}=2 T_{\mathrm{c}}$, thereby avoiding unnecessary updates of $v_{i}$ at high $T$. This procedure for setting $\alpha$ also requires rescaling of the costs; for all problems we set $c_{i} \rightarrow c_{i} / \max _{j}\left(c_{j}\right)$. See Table 1 for a summary of the parameters used. The evolution of the MF variables $\left\{v_{i}\right\}$ is conveniently monitored by the saturation $\Sigma$,

Table 1

Summary of the parameters $k, \alpha$ and $T_{0}$ used in the algorithm

\begin{tabular}{llll}
\hline & Unicost SCP & \multicolumn{2}{l}{ Non-unicost SCP } \\
\cline { 3 - 4 } & & Prerun & Second run \\
\hline$k$ & 0.80 & 0.65 & 0.8 \\
$\alpha$ & 0.5 & 1.01 & $1.05 * c_{\max }^{*}$ \\
$T_{0}$ & 50 & 50 & $2 * T_{\mathrm{c}}$ \\
\hline
\end{tabular}

$\Sigma=\frac{4}{N} \sum_{i=1}^{N}\left(v_{i}-1 / 2\right)^{2}$

A completely "undecided" configuration, $\Sigma=0$, means that every $v_{i}$ has the value $1 / 2$. During the annealing process, as each $v_{i}$ approaches either 1 or $0, \Sigma$ converges to 1 . The transition between $\Sigma=0$ and $\Sigma=1$ is usually smooth for a generic SCP. However, when a bifurcation is encountered (see above), $\Sigma$ can change abruptly; this occurs, e.g., for unicost SCP where there is no natural ordering among the costs $\left\{c_{i}\right\}$.

Fig. 1 shows the evolution of the MF variables $\left\{v_{i}\right\}$ and the saturation $\Sigma$ for the problems 4.1 and CYC. 9 (see Appendix C, Tables 3 and 4); the latter is a unicost problem, and as can be seen from Fig. 1b, it clearly exhibits a bifurcation.

A summary of the algorithm can be found in Appendix B, while Appendix D gives the address of and instructions for a WWW server, where the MF algorithm is applied to user-defined SCPs.

\subsection{Numerical results}

The performance of the algorithm is evaluated using 16 problem sets found in the OR-Library benchmark database [15]. These 16 sets consist of 91 problems, out of which 19 are unicost SCP. The algorithm is coded in $\mathrm{C}$ and the computations are done on a $400 \mathrm{MHz}$ Pentium II PC. The details of the OR-library problems are given in Table 3 and our results can be found in Tables 4-6 in Appendix C. The optimal or currently best known values are taken from Refs. [1-3,5,8,12].

For each of the test SCPs, 10 trials of our algorithm are performed. In Tables 4-6, the best and the average costs are listed for each problem. For 10 of the problems our method found the optimal solution. The MF results are typically within a few percent of the optimal solutions, as can be seen in Table 2. Large relative deviations from optimum are seen in the unicost CLR problems (see Appendix C, Tables 3 and 6), which appear to be difficult for our approach. However, the optimal (integer) costs for these problems are low (23-26); this gives a large effect on the relative deviations 

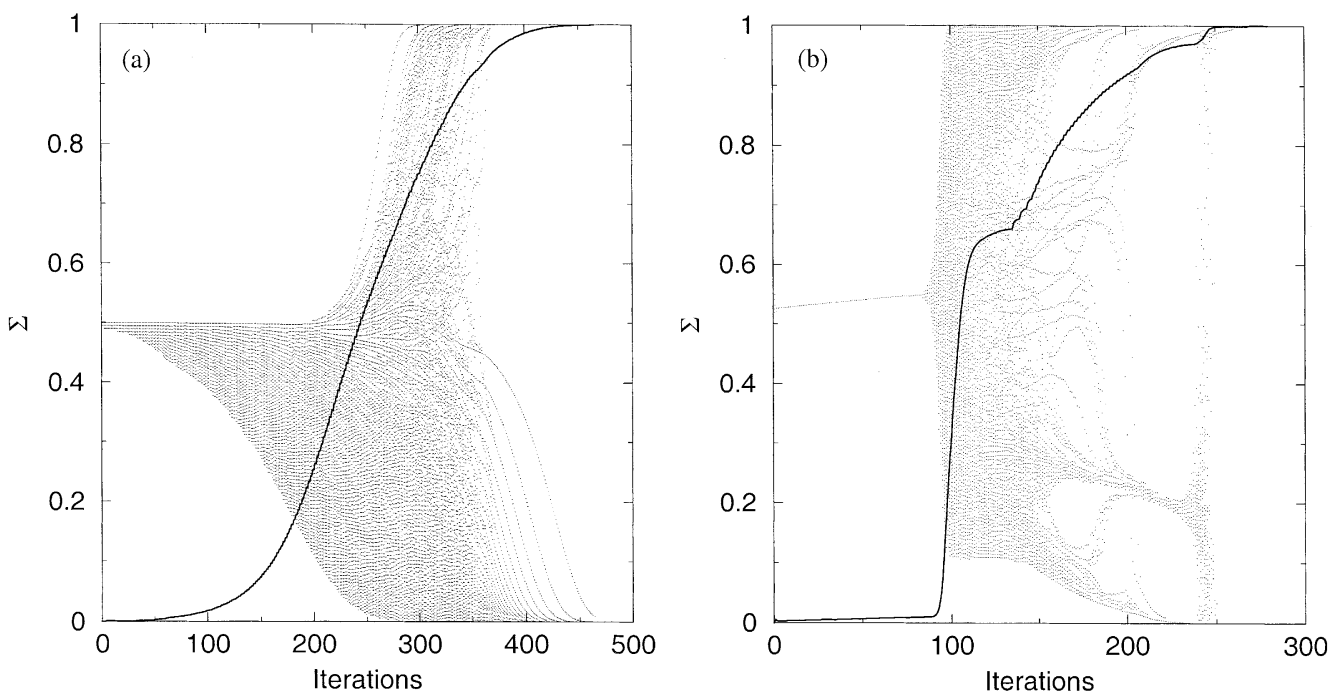

Fig. 1. Evolution of the MF variables $v_{i}$ as $T$ is lowered for 4.1 (a) and CYC. 9 (b), respectively. Also shown is $\Sigma$ (Eq. (18)). Note that the number of iterations is intentionally large for visualization purposes.

Table 2

Mean relative deviation from the optimal (or best known) solution and normalized CPU times for the MF method and other approaches $^{\mathrm{a}}$

\begin{tabular}{|c|c|c|c|c|c|c|c|c|c|c|c|c|}
\hline \multirow[t]{2}{*}{ Problem set } & \multicolumn{6}{|c|}{ Rel. deviation (\%) } & \multicolumn{6}{|c|}{ (Normalized) CPU Time } \\
\hline & $\overline{\mathrm{MF}}$ & GA & $\mathrm{R}-\mathrm{Gr}$ & Alt-Gr & $\mathrm{BB}$ & $\mathrm{LH}$ & $\overline{\mathrm{MF}}$ & GA & $\mathrm{R}-\mathrm{Gr}$ & Alt-Gr & BB & $\mathrm{LH}$ \\
\hline 4 & 2.1 & 0 & - & - & - & - & 1 & 11 & - & - & - & - \\
\hline 5 & 2.7 & 0.09 & - & - & - & - & 1 & 3.6 & - & - & - & - \\
\hline 6 & 3.5 & 0 & - & - & - & - & 1 & 3.9 & - & - & - & - \\
\hline A & 2.2 & 0 & - & - & - & - & 1 & 4.6 & - & - & - & - \\
\hline $\mathrm{B}$ & 1.1 & 0 & - & - & - & - & 1 & 2.9 & - & - & - & - \\
\hline $\mathrm{C}$ & 1.7 & 0 & - & - & - & - & 1 & 3.9 & - & - & - & - \\
\hline $\mathrm{D}$ & 2.9 & 0 & - & - & - & - & 1 & 2.5 & - & - & - & - \\
\hline NRE & 3.5 & 0 & - & - & - & - & 1 & 1.1 & - & - & - & - \\
\hline NRF & 4.4 & 0 & - & - & - & - & 1 & 0.32 & - & - & - & - \\
\hline NRG & 3.1 & 0 & - & - & - & - & 1 & 3.4 & - & - & - & - \\
\hline $\mathrm{NRH}$ & 2.0 & 0 & - & - & - & - & 1 & 2.5 & - & - & - & - \\
\hline Rail & 2.0 & - & - & - & - & 2.0 & 1 & - & - & - & - & 3.7 \\
\hline \multicolumn{13}{|c|}{ Unicost problems } \\
\hline $\mathrm{E}$ & 0 & - & 0 & 0 & - & - & 1 & - & $\mathrm{b}$ & $\mathrm{b}$ & - & - \\
\hline CYC & 6.8 & - & 13 & 3.0 & - & - & 1 & - & 10 & 0.21 & - & - \\
\hline CLR & 16 & - & 13 & 22 & - & - & 1 & - & 0.98 & 0.57 & - & - \\
\hline STS & 2.5 & - & - & - & 0 & - & 1 & - & - & - & $8 \cdot 10^{4}$ & - \\
\hline
\end{tabular}

${ }^{\mathrm{a}}$ The results for the genetic algorithm (GA) are taken from [2]. R-Gr and Alt-Gr are greedy heuristics algorithms from [5]. BB is a branch and bound algorithm and LH is a Lagrangian based heuristic, with results taken from [12,3], respectively.

${ }^{\mathrm{b}}$ The CPU times reported for these problems was 0.0 and a comparison is therefore not possible.

even for a small change in the found costs. Decreasing the obtained costs by unity will change the mean relative deviation from $16 \%$ to $10 \%$. It is also important to notice that we use a common set of algorithm parameters $\left(\alpha, k, T_{0}\right)$ for all unicost problems, without any parameter optimization for 
Table 3

Test problem details ${ }^{\mathrm{a}, \mathrm{b}}$

\begin{tabular}{|c|c|c|c|c|c|}
\hline Problem set & $\begin{array}{l}\text { Rows } \\
(M)\end{array}$ & $\begin{array}{l}\text { Columns } \\
(N)\end{array}$ & $\begin{array}{l}\text { Density } \\
(\%)\end{array}$ & $\begin{array}{l}\text { Number of ones per row } \\
{[\mathrm{max}-\mathrm{min}-\text { ave }]}\end{array}$ & Number of problems \\
\hline 4 & 200 & 1000 & 2 & $36-8-20$ & 10 \\
\hline 5 & $"$ & 2000 & 2 & $60-21-40$ & 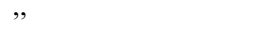 \\
\hline 6 & $"$ & 1000 & 5 & $71-29-50$ & 5 \\
\hline A & 300 & 3000 & 2 & $81-38-60$ & $"$ \\
\hline B & ", & $"$ & 5 & $191-114-150$ & $"$ \\
\hline $\mathrm{C}$ & 400 & 4000 & 2 & $105-56-80$ & $"$ \\
\hline $\mathrm{D}$ & $"$ & $"$ & 5 & $244-159-200$ & $"$ \\
\hline NRE & 500 & 5000 & 10 & $561-444-499$ & $"$ \\
\hline $\mathrm{NRF}$ & $"$ & $"$ & 20 & 1086-914-999 & $"$ \\
\hline NRG & 1000 & 10000 & 2 & $258-153-199$ & " \\
\hline $\mathrm{NRH}$ & $"$ & $"$ & 5 & $580-436-499$ & $"$ \\
\hline Rail.507 & 507 & 63009 & 1.3 & $7753-1-807$ & 1 \\
\hline Rail.516 & 516 & 47311 & 1.3 & $7805-1-610$ & $"$ \\
\hline Rail.582 & 582 & 55515 & 1.2 & 8919-1-690 & $"$ \\
\hline Rail.2536 & 2536 & 1081841 & 0.40 & $86666-1-4335$ & $"$ \\
\hline Rail.2586 & 2586 & 920683 & 0.34 & $72553-1-3097$ & $"$ \\
\hline Rail.4284 & 4284 & 1092610 & 0.24 & $56181-1-2633$ & $"$ \\
\hline Rail.4872 & 4872 & 968672 & 0.20 & 69708-1-1897 & $"$ \\
\hline \multicolumn{6}{|c|}{ Unicost problems } \\
\hline $\mathrm{E}$ & 50 & 500 & 20 & $124-77-100$ & 5 \\
\hline CYC.6 & 240 & 192 & 2.1 & 4-4-4 & 1 \\
\hline CYC.7 & 672 & 448 & 0.9 & " & $"$ \\
\hline CYC.8 & 1792 & 1024 & 0.4 & $"$ & $"$ \\
\hline CYC.9 & 4608 & 2304 & 0.2 & $"$ & $"$ \\
\hline CYC.10 & 11520 & 5120 & 0.08 & " & $"$ \\
\hline CYC.11 & 28160 & 11264 & 0.04 & $"$ & $"$ \\
\hline CRL.10 & 511 & 210 & 12 & $126-10-26$ & $"$ \\
\hline CRL.11 & 1023 & 330 & $"$ & $210-20-41$ & $"$ \\
\hline CRL.12 & 2047 & 495 & $"$ & $330-30-62$ & " \\
\hline CRL.13 & 4095 & 715 & ” & $495-50-89$ & " \\
\hline STS.45 & 330 & 45 & 6.7 & $3-3-3$ & $"$ \\
\hline STS.81 & 1080 & 81 & 3.7 & ", & $"$ \\
\hline STS.135 & 3015 & 135 & 2.2 & $"$ & " \\
\hline STS.243 & 9801 & 243 & 1.2 & " & $"$ \\
\hline
\end{tabular}

${ }^{\text {a }}$ The density refers to the percentage of ones in the $A$ matrix.

${ }^{\mathrm{b}}$ The maximum, minimum and average of ones per row is taken over all problems in each set.

each problem. Another set of parameters might be more advantageous for the CLR problems.

In our implementation of the MF algorithm, the time $\tau$ for a complete update of all variables $\left\{v_{i}\right\}$ scales approximately linearly with the number of non-zero entries in $A$,

$\tau \propto N M \rho$.

This appealing property is feasible due to efficient calculations of $\Delta E_{i}$ in Eq. (14) that utilizes the sparse nature of $A$. For the total solution time, $\tau$ should be multiplied by the number of iterations needed - empirically around 100 , independently of problem size. Fig. 2 shows the mean solution time versus $N M \rho$.

Compared to other heuristic approaches, ours does not find the optimal cost as often as, e.g., the genetic algorithm [2]. It is however very competitive with respect to speed. Table 2 shows a more detailed comparison with the genetic algorithm. In order to compare CPU times for different com- 

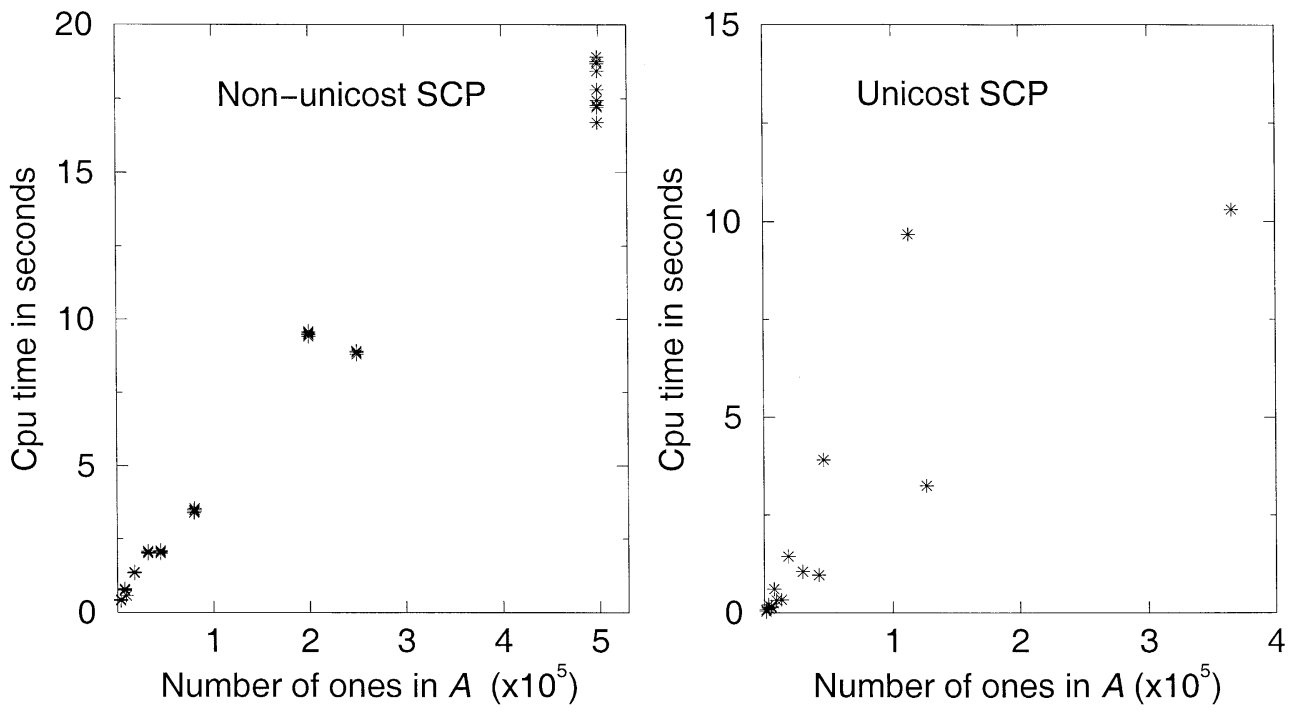

Fig. 2. Solution time in seconds, referring to a Pentium II $400 \mathrm{MHz}$ computer, as function of the number of non-zero entries in the problem matrix $A$. Left and right figure shows non-unicost and unicost SCP, respectively. The rail problems are not shown since a slightly different implementation is used for these problems (see Appendix B for details).

puters we have used the Linpack benchmark [4] as a reference. In Ref. [5] nine different approximation algorithms were tested on a large number of unicost problems (including the set considered here); our approach is comparable to the top ones in both performance and speed (see Table 2).

It is worth noting that for one of the small Rail problems (Rail. 516) we found a cover with the cost 186, which is smaller than the lower bound reported in [3].

\section{Summary}

We have developed a MF feedback neural network approach for solving SCPs. The method is applied to a standard set of benchmark problems available in the OR-library database. The method is also implemented in a public WWW server.

The inequality constraints involved are conveniently handled by means of a multi-linear penalty function that fits nicely into the MF framework. The bifurcation structure of the MF dynamics involved in the neural network approach is analyzed by means of a linearized dynamics. A simple and self-contained derivation of the MF equations is provided.

High quality solutions are consistently found throughout a range of problem sizes ranging up to $5 \times 10^{3}$ rows and $10^{6}$ columns for the OR-library problems without having to fine-tune the parameters, with a time consumption scaling as the number of non-zero matrix elements. The approach is extremely efficient, typically requiring a few seconds on a Pentium II $400 \mathrm{MHz}$ computer.

The MF approach to SCP can easily be modified to apply to the related, and more constrained, set partitioning problem. One simply has to replace the inequality constraint term with one that handles the equality constraint present in the set partitioning problem.

\section{Acknowledgements}

This work was in part supported by the Swedish Natural Science Research Council, the Swedish Board for Industrial and Technical Development and the Swedish Foundation for Strategic Research. 


\section{Appendix A. Mean field approximation}

Here follows for completeness a derivation of the MF equations (see, e.g., [16]). Let $E(\mathscr{S})$ be an energy function of a set of binary decision variables (spins) $\mathscr{S}=\left\{s_{i} \mid s_{i} \in\{0,1\}, i=1, \ldots, N\right\}$. If we assume a Boltzmann probability distribution for the spins, the average $\left\langle s_{i}\right\rangle_{T}$ will be given by

$$
\left\langle s_{i}\right\rangle_{T}=\frac{\sum_{\mathscr{S}} s_{i} \exp (-E(\mathscr{S}) / T)}{\sum_{\mathscr{S}} \exp (-E(\mathscr{S}) / T)}
$$

where the sums run over all possible configurations $\mathscr{S}$. We can manipulate this expression to obtain,

$$
\left\langle s_{i}\right\rangle_{T}=\frac{\left.\sum_{\mathscr{S}} \exp (-E(\mathscr{S}) / T) \frac{\sum_{s_{i}=0,1} s_{i} \exp \left(-E\left(\mathscr{S}^{(i)}, s_{i}\right) / T\right)}{\sum_{s_{i}=0,1} \exp \left(-E\left(\mathscr{S}^{(i)}, s_{i}\right) / T\right.}\right)}{\sum_{\mathscr{S}} \exp (-E(S) / T)},
$$

where $\mathscr{S}^{(i)}$ denotes the set $\left\{s_{j}, j \neq i\right\}$ of all spins but $s_{i}$. If we now perform the sums over $s_{i}$ in the numerator, we get

$$
\begin{aligned}
\left\langle s_{i}\right\rangle_{T} & =\frac{\sum_{\mathscr{S}} \exp (-E(\mathscr{S}) / T) \frac{1}{1+\exp \left(\Delta E_{i} / T\right)}}{\sum_{\mathscr{S}} \exp (-E(\mathscr{S}) / T)} \\
& \equiv\left\langle\frac{1}{1+\exp \left(\Delta E_{i} / T\right)}\right\rangle_{T},
\end{aligned}
$$

where

$$
\Delta E_{i}\left(\mathscr{S}^{(i)}\right)=E\left(\mathscr{S}^{(i)}, s_{i}=1\right)-E\left(\mathscr{S}^{(i)}, s_{i}=0\right) .
$$

So far there are no approximations, the expression for $\left\langle s_{i}\right\rangle_{T}$ has just been rewritten. Eq. (A.3) states that the expectation value of $s_{i}$ is equal to the expectation value of a nonlinear function $f$ of all the other spins. The mean field approximation consists of approximating the expectation value $\left\langle f\left(\mathscr{S}^{(i)}\right)\right\rangle$ by $f\left(\left\langle\mathscr{S}^{(i)}\right\rangle\right)$. With $v_{i}$ denoting $\left\langle s_{i}\right\rangle$, and $\mathscr{V}^{(i)}$ the complementary set $\left\{v_{j}, j \neq i\right\}$, this amounts to making the replacement

$$
\begin{aligned}
& \left\langle\frac{1}{1+\exp \left(\Delta E_{i}\left(\mathscr{S}^{(i)}\right) / T\right)}\right\rangle_{T} \\
& \rightarrow \frac{1}{1+\exp \left(\Delta E_{i}\left(\mathscr{V}^{(i)}\right) / T\right)}
\end{aligned}
$$

in Eq. (A.3).

\footnotetext{
1. Rescale all weights such that $c_{j} \in[0,1] \quad j=1, \ldots, N$

2. Initiate all $v_{j}$ 's close to 0.5 $\left(v_{j} \in[0.499,0.501]\right.$ uniformly random)
}

3. Set $\alpha=1.05 c_{\max }$, or $\alpha=0.5$ for unicost problems

4. Set the temperature $T=2 T_{c}$, or $T=50$ for unicost problems

5. Randomly (without replacement) select one variable $v_{k}$

6. Update $v_{k}$ according to Eq. (12)

7. Repeat points 5.-6. $N$ times (so that all $v_{j}$ have been updated once)

8. Repeat points 5.-7. until no changes occur (e.g. defined by $1 / N \sum_{j}^{N}\left|v_{j}-v_{j}^{(\text {old })}\right| \leq 0.01$ )

9. Decrease the temperature, $T \rightarrow 0.80 T$

10. Repeat 5-9 until $\Sigma$ (Eq. (18)) is close to 1 (e.g. $\Sigma \geq(N-0.5) / N)$

11. Finally, the mean field solution is given by setting $s_{j}=1$ if $v_{j} \geq 0.5$ and $s_{j}=0$ otherwise, $j=1, \ldots, N$

Fig. 3. Implementation details of the MF algorithm for SCPs. 
This results in a set of self-consistency equations for $\mathscr{V}$, the MF equations

$v_{i}=\frac{1}{1+\exp \left(\Delta E_{i}\left(\mathscr{V}^{(i)}\right) / T\right)}, \quad i=1, \ldots, N$, which in general must be solved numerically, e.g., by iteration.

The MF approximation often becomes exact in the limit of infinite range interactions where each

Table 4

Results for problems 4-D (see Table 3$)^{\mathrm{a}}$

\begin{tabular}{|c|c|c|c|c|}
\hline Problem & Optimal value & $\begin{array}{l}\text { Best MF solution } \\
\text { in } 10 \text { trials }\end{array}$ & $\begin{array}{l}\text { Average MF solution } \\
\text { over } 10 \text { trials }\end{array}$ & Solution time \\
\hline 4.1 & 429 & 435 & 435.6 & 0.44 \\
\hline 4.2 & 512 & 517 & 518.0 & 0.49 \\
\hline 4.3 & 516 & 531 & 532.7 & 0.45 \\
\hline 4.4 & 494 & 512 & 520.9 & 0.48 \\
\hline 4.5 & 512 & 522 & 524.1 & 0.46 \\
\hline 4.6 & 560 & 566 & 567.8 & 0.44 \\
\hline 4.7 & 430 & 446 & 446.0 & 0.45 \\
\hline 4.8 & 492 & 492 & 493.8 & 0.46 \\
\hline 4.9 & 641 & 658 & 661.4 & 0.47 \\
\hline 4.10 & 514 & 521 & 521.0 & 0.45 \\
\hline 5.1 & 253 & 260 & 268.6 & 0.87 \\
\hline 5.2 & 302 & 316 & 316.0 & 0.83 \\
\hline 5.3 & 226 & 229 & 229.0 & 0.86 \\
\hline 5.4 & 242 & 247 & 247.5 & 0.86 \\
\hline 5.5 & 211 & 214 & 214.3 & 0.83 \\
\hline 5.6 & 213 & 213 & 213.2 & 0.82 \\
\hline 5.7 & 293 & 304 & 305.0 & 0.86 \\
\hline 5.8 & 288 & 299 & 300.1 & 0.90 \\
\hline 5.9 & 279 & 281 & 281.0 & 0.82 \\
\hline 5.10 & 265 & 273 & 274.0 & 0.83 \\
\hline 6.1 & 138 & 143 & 143.0 & 0.63 \\
\hline 6.2 & 146 & 153 & 153.2 & 0.62 \\
\hline 6.3 & 145 & 150 & 150.2 & 0.60 \\
\hline 6.4 & 131 & 132 & 133.1 & 0.62 \\
\hline 6.5 & 161 & 169 & 169.8 & 0.62 \\
\hline A.1 & 253 & 260 & 261.5 & 1.5 \\
\hline A. 2 & 252 & 257 & 258.3 & 1.5 \\
\hline A. 3 & 232 & 238 & 241.3 & 1.5 \\
\hline A. 4 & 234 & 238 & 239.7 & 1.5 \\
\hline A. 5 & 236 & 238 & 238.9 & 1.4 \\
\hline B. 1 & 69 & 70 & 71.2 & 2.2 \\
\hline B. 2 & 76 & 77 & 77.6 & 2.3 \\
\hline B. 3 & 80 & 83 & 83.7 & 2.2 \\
\hline B. 4 & 79 & 80 & 80.0 & 2.3 \\
\hline B. 5 & 72 & 72 & 72.0 & 2.3 \\
\hline C. 1 & 227 & 233 & 233.6 & 2.2 \\
\hline C. 2 & 219 & 222 & 224.3 & 2.2 \\
\hline C. 3 & 243 & 249 & 251.1 & 2.3 \\
\hline C. 4 & 219 & 220 & 220.1 & 2.3 \\
\hline C. 5 & 215 & 219 & 219.1 & 2.2 \\
\hline D. 1 & 60 & 64 & 64.6 & 3.7 \\
\hline D. 2 & 66 & 66 & 66.3 & 3.8 \\
\hline D. 3 & 72 & 73 & 75.1 & 3.9 \\
\hline D. 4 & 62 & 63 & 63.0 & 3.8 \\
\hline D. 5 & 61 & 64 & 64.6 & 3.8 \\
\hline
\end{tabular}

${ }^{\mathrm{a}}$ Solution time refers to $400 \mathrm{MHz}$ Pentium II CPU seconds and includes the prerun for non-unicost problems. 
spin variable interacts with all the others. This can be seen from $\Delta E_{i}\left(\mathscr{S}^{(i)}\right)$ which then becomes a sum of many (approximately) independent random numbers, and a central limit theory can be applied.

\section{Appendix B. Algorithm details}

Here follows a summary of the MF annealing algorithm for finding approximate solutions to SCPs. The procedure presented below is used for all problems in this study except for the large rail problems where numerical problems caused by limited machine precision comes into play. The problem arises when calculating the product in Eq. (14), which for large problems can contain many factors. A work-around is implemented by a simple truncation,

$v_{i}:= \begin{cases}0 & \text { if } v_{i}<0.05, \\ v_{i} & \text { otherwise. }\end{cases}$

This numerical fix is only used when calculating the $\Delta E_{i}\left(\mathscr{V}^{(i)}\right)$ in Eq. (14).

Algorithmic outline of our approach is shown in Fig. 3.

\section{Appendix C. Benchmark results}

The outline of this appendix is as follows: Table 3 lists the properties of each problem set in terms of size, density of ones in the $A$ matrix, rows

Table 5

Results for problems NRE-Rail (see Table 3) ${ }^{\mathrm{a}}$

\begin{tabular}{|c|c|c|c|c|}
\hline Problem & Current best value & $\begin{array}{l}\text { Best MF solution } \\
\text { in } 10 \text { trials }\end{array}$ & $\begin{array}{l}\text { Average MF solution } \\
\text { over } 10 \text { trials }\end{array}$ & Solution time \\
\hline NRE. 1 & 29 & 29 & 29.5 & 9.8 \\
\hline NRE. 2 & 30 & 32 & 32.1 & 9.8 \\
\hline NRE.3 & 27 & 28 & 28.2 & 9.7 \\
\hline NRE.4 & 28 & 29 & 29.7 & 9.8 \\
\hline NRE.5 & 28 & 29 & 29.0 & 9.8 \\
\hline NRF.1 & 14 & 14 & 14.9 & 19 \\
\hline NRF. 2 & 15 & 15 & 15.4 & 18 \\
\hline NRF.3 & 14 & 15 & 15.2 & 19 \\
\hline NRF.4 & 14 & 15 & 15.4 & 19 \\
\hline NRF.5 & 13 & 14 & 14.7 & 19 \\
\hline NRG.1 & 176 & 180 & 180.1 & 10 \\
\hline NRG. 2 & 155 & 157 & 159.0 & 10 \\
\hline NRG.3 & 166 & 173 & 174.9 & 10 \\
\hline NRG.4 & 168 & 175 & 176.3 & 10 \\
\hline NRG.5 & 168 & 175 & 176.9 & 11 \\
\hline NRH.1 & 64 & 65 & 66.4 & 21 \\
\hline NRH. 2 & 64 & 66 & 67.0 & 21 \\
\hline NRH.3 & 59 & 62 & 62.8 & 20 \\
\hline NRH.4 & 58 & 60 & 61.8 & 21 \\
\hline NRH. 5 & 55 & 56 & 56.4 & 21 \\
\hline Rail.507 & 174 & 187 & 188.2 & 37 \\
\hline Rail.516 & 211 & 186 & 187.9 & 26 \\
\hline Rail.582 & 182 & 222 & 225.5 & 32 \\
\hline Rail.2536 & 691 & 737 & 740.0 & 1100 \\
\hline Rail.2586 & 951 & 1018 & 1026.7 & 830 \\
\hline Rail.4284 & 1065 & 1152 & 1162.1 & 1100 \\
\hline Rail.4872 & 1534 & 1640 & 1643.5 & 1050 \\
\hline
\end{tabular}

${ }^{\text {a }}$ Solution time refers to $400 \mathrm{MHz}$ Pentium II CPU seconds and includes the prerun for non-unicost problems. The notation current best value may for some instances mean optimal value. 
Table 6

Results for the unicost problems E - STS (see Table 3) ${ }^{\mathrm{a}}$

\begin{tabular}{lrccc}
\hline Problem & Current best value & $\begin{array}{l}\text { Best MF solution } \\
\text { in } 10 \text { trials }\end{array}$ & $\begin{array}{c}\text { Average MF solution } \\
\text { over } 10 \text { trials }\end{array}$ & $\begin{array}{c}\text { Solution time } \\
\text { E.1 }\end{array}$ \\
E.2 & 5 & 5 & 5.3 & 0.15 \\
E.3 & 5 & 5 & 5.0 & 0.14 \\
E.4 & 5 & 5 & 5.0 & 0.15 \\
E.5 & 5 & 5 & 5.0 & 0.14 \\
CYC.6 & 5 & 5 & 5.0 & 0.16 \\
CYC.7 & 60 & 62 & 63.0 & 0.08 \\
CYC.8 & 144 & 151 & 153.4 & 0.20 \\
CYC.9 & 344 & 348 & 352.1 & 0.62 \\
CYC. 10 & 780 & 829 & 832.6 & 1.6 \\
CYC.11 & 1792 & 1870 & 1882.3 & 3.9 \\
CLR.10 & 4103 & 4240 & 4248.7 & 9.6 \\
CLR.11 & 25 & 27 & 29.0 & 0.36 \\
CLR.12 & 23 & 26 & 28.9 & 1.0 \\
CLR.13 & 26 & 30 & 30.9 & 3.2 \\
STS.45 & 26 & 31 & 32.9 & 10 \\
STS.81 & 30 & 31 & 31.8 & 0.03 \\
STS.135 & 61 & 63 & 63.9 & 0.11 \\
STS.243 & 104 & 105 & 107.4 & 0.32 \\
\hline
\end{tabular}

${ }^{a}$ Solution time refers to $400 \mathrm{MHz}$ Pentium II CPU seconds. The notation current best value may for some instances mean optimal value.

sums and finally the number of sub-problems in each set. Tables 4-6 contain the results found using our algorithm on each of the sub-problems in each problem set.

\section{Appendix D. WWW server}

A program executing the MF algorithm for set covering problems as presented in this paper can be publicly used by means of a World Wide Web server. A user can interactively submit a file defining an instance of SCP, and obtain the found solution. The URL of the WWW server is:

\section{http://www.thep.lu.se/complex/ \\ mf_server.html}

An instance of SCP is defined by specifying the costs $c_{i}$ and the matrix $A$. Two formats, row and column ordering, are supported for the file that lists $c_{i}$ and the none-zero entries of $A$; they are defined as follows:
Row ordering

$M N$

$c_{1} c_{2} \ldots c_{N}$

$\hat{M}_{1}$ space separated list of all non-zero entries for row 1

$\hat{M}_{2}$ space separated list of all non-zero entries for row 2

$\ddot{\hat{M}}_{M} \quad$ space separated list of all non-zero entries for row $M$

Column ordering

$M N$

$c_{1} \hat{N}_{1} \quad$ space separated list of all non-zero entries for column 1

$c_{2} \hat{N}_{2} \quad$ space separated list of all non-zero entries for column 2

...

$c_{N} \hat{N}_{N} \quad$ space separated list of all non-zero entries for column $N$

The column sums $\hat{N}_{i}$ and row sums $\hat{M}_{k}$ are defined in Eqs. (7) and (8). As an example, consider the SCP instance defined by 
$\vec{c}=(1,2,3,4,5), \quad A=\left(\begin{array}{ccccc}1 & 0 & 1 & 0 & 1 \\ 0 & 1 & 0 & 1 & 0 \\ 1 & 1 & 0 & 0 & 1 \\ 0 & 0 & 1 & 1 & 1\end{array}\right)$

for which the column and row ordering formats read

\begin{tabular}{|c|c|}
\hline Row ordering & Column ordering \\
\hline 45 & 45 \\
\hline 12345 & 1213 \\
\hline 3135 & 2223 \\
\hline 224 & 3214 \\
\hline 3125 & 4224 \\
\hline 3345 & 53134 \\
\hline
\end{tabular}

The solver returns the found cost (energy) $E$ (Eq. (3)), together with a characterization of the problem. Upon request, a file that lists the columns used in the solution is also provided.

There is a limitation $(B)$ on the size of the problems that can be submitted to the server. Instances of SCP with $M N \rho>B$ will not be considered. Presently $B$, which is limited by the available memory of the server, is given by $3 \times 10^{6}$.

\section{References}

[1] J.E. Beasley, An algorithm for the set covering problem, European Journal of Operational Research 31 (1987) 8593.

[2] J.E. Beasley, A genetic algorithm for the set covering problem, European Journal of Operational Research 94 (1994) 392-404.
[3] S. Ceria, P. Nobili, A. Sassano, A Lagrangian-based heuristics for large scale set-covering problems, Mathematical Programming 81 (1998) 215-228.

[4] J.J. Dongarra, Performance of various computers using standard linear equations software, http://http://www.netlib.org/benchmark/performance.ps.

[5] T. Grossmann, A. Wool, Computational experience with approximation algorithms for the set covering algorithm, European Journal of Operational Research 101 (1987) 8192.

[6] J.J. Hopfield, D.W. Tank, Neural computation of decisions in optimization problems, Biological Cybernetics 52 (1985) 141-152.

[7] J. Häkkinen, M. Lagerholm, C. Peterson, B. Söderberg, A Potts neuron approach to communication routing, Neural Computation 10 (1998) 1587-1599.

[8] N. Karmarkar, M.G.C. Resende, K.G. Ramakrishnan, An interior point algorithm to solve computationally difficult set covering problems, Mathematical Programming 52 (1991) 597-618.

[9] S. Kirkpatrick, C.D. Gelatt, M.P. Vecchi, Optimization by simulated annealing, Science 220 (1983) 671-680.

[10] M. Lagerholm, C. Peterson, B. Söderberg, Airline crew scheduling with Potts neurons, Neural Computation 9 (1997) 1589-1599.

[11] M. Lagerholm, C. Peterson, B. Söderberg, Airline crew scheduling using Potts mean field techniques, European Journal of Operational Research 120 (2000) 81-96.

[12] C. Mannino, A. Sassano, Solving hard set covering problems, Operations Research Letters 18 (1995) 1-5.

[13] M. Ohlsson, H. Pi, A study of the mean field approach to knapsack problems, Neural Networks 10 (1997) 263-271.

[14] M. Ohlsson, C. Peterson, B. Söderberg, Neural networks for optimization problems with inequality constraints - the knapsack problem, Neural Computation 5 (1993) 331-339.

[15] The OR-Library, http://www.ms.ic.ac.uk/info.html.

[16] G. Parisi, Statistical Field Theory, Addison-Wesley, New York, 1988.

[17] C. Peterson, B. Söderberg, A new method for mapping optimization problems onto neural networks, International Journal of Neural Systems 1 (1989) 3-22. 